\title{
Feasible Generalized Stein-Rule Restricted Ridge Regression Estimators
}

\author{
N. ÖZBAY, I. DAWOUD AND S. KAÇIRANLAR
}

\begin{abstract}
Several versions of the Stein-rule estimators of the coefficient vector in a linear regression model are proposed in the literature. In the present paper, we propose new feasible generalized Stein-rule restricted ridge regression estimators to examine multicollinearity and autocorrelation problems simultaneously for the general linear regression model, when certain additional exact restrictions are placed on these coefficients. Moreover, a Monte Carlo simulation experiment is performed to investigate the performance of the proposed estimator over the others.
\end{abstract}

Keywords: Feasible Estimator; Stein-rule Estimator; Restricted Ridge Estimator; Multicollinearity; Autocorrelation

\section{INTRODUCTION}

Stein estimator is proposed by Stein (1956). The family of Stein-rule (SR) estimators proposed by James and Stein (1960) has smaller variability than the ordinary least squares (OLS) estimator under quadratic risk with a simple condition that the number of explanatory variables are more than two. For the coefficient vector in a linear regression model with spherical disturbances, SR estimators have been extensively used. The comparisons under different loss functions and several versions of the SR estimators have also received considerable attention in the literature. Chaturvedi and Shukla (1990) consider a SR estimator for estimating the regression model with autocorrelated errors and derive its asymptotic properties. Wan and Chaturvedi (2000) extend the analysis of Chaturvedi and Shukla (1990) to a class of operational variants of the minimum mean squared error estimators. Srivastava and Srivastava $(1983,1984)$ and Srivastava and Chandra (1991) consider families of improved restricted estimators obtained by mixing SR with restricted least squares. Chaturvedi et al. (1996) extends the analysis of Srivastava and Srivastava (1984) to models with nonspherical disturbances. Chaturvedi et al. (2001) 
propose a SR estimator for the general linear regression model with nonspherical disturbances and a set of linear restrictions binding the regression coefficients.

Many authors have studied the predicted performance of predictors obtained by using different estimators for either the actual values or the average values of the study variable at a time. Toutenburg and Shalabh (1996) analyzed the performance properties of predictors arising from the methods of restricted regression and mixed regression besides least squares according to the target function. Then, Toutenburg and Shalabh (2000) considered the family of SR estimators proposed by Srivastava and Srivastava (1983) and analyzed performance properties of this family when the objective is to predict values outside the sample and within the sample. Kumar et al. (2008) considered two families of SR estimators proposed by Srivastava and Srivastava (1984) and analyzed performance properties of these families under the target function. Shalabh, Toutenburg and Heumann (2009) introduced the extended balanced loss function (EBLF) under the target function and discussed the SR estimation. Moreover, Chaturvedi and Shalabh (2014) discussed the bayesian estimation of regression coefficients under the EBLF.

In the present paper, we extend the idea of Chaturvedi et al. (2001) to models under multicollinearity and propose new families of feasible generalized SR restricted ridge regression (FGSRR) estimator for the general linear regression model with autocorrelation, multicollinearity and a set of linear restrictions binding the regression coefficients, in Section 2. In Section 3, we indicate the prediction mean square error (PMSE) under the target function criterion. Moreover, we perform a Monte Carlo simulation experiment to compare the performance of the new estimators to the others in terms of the PMSE criterion under the target function in Section 4. 


\section{THE MODEL AND ESTIMATORS}

Consider the following linear regression model

$$
Y=X \beta+\varepsilon, \varepsilon \sim\left(0, \sigma^{2} \mathrm{I}_{n}\right)
$$

where $Y$ is an $n \times 1$ vector of observations on the dependent variable, $X$ is an $n \times p$ known design matrix of rank $p, \beta$ is an $p \times 1$ vector of unknown parameters, and $\varepsilon$ is an $\mathrm{n} \times 1$ vector of random errors with zero mean and variance $\sigma^{2} I_{n}$, where $I_{n}$ is an identity matrix of order $n$.

The OLS estimator of $\beta$ is defined as:

$$
\hat{\beta}=\left(X^{\prime} X\right)^{-1} X^{\prime} Y
$$

Both the OLS estimator and its covariance matrix heavily depend on the characteristics of the $X^{\prime} X$ matrix. If $X^{\prime} X$ is ill-conditioned, i.e. the column vectors of $X$ are linearly dependent, the OLS estimator is sensitive to a number of errors. One of the most popular estimator dealing with multicollinearity is the ordinary ridge regression (ORR) estimator proposed by Hoerl and Kennard (1970a) and is defined as

$$
\hat{\beta}_{k}=\left(X^{\prime} X+k I_{p}\right)^{-1} X^{\prime} Y=\left(I_{p}+k\left(X^{\prime} X\right)^{-1}\right)^{-1} \hat{\beta},
$$

where the constant $k>0$ is known as the biasing parameter.

One may often have some exact linear restrictions binding the regression coefficients. Hence, we assume the exact linear restrictions binding the regression coefficients vector $\beta$ of $m$ linearly independent rows as follows:

$$
r=R \beta
$$

where $r$ is an $m \times 1$ vector and $R$ is an $m \times p$ matrix of rank $m(<p)$. The wellknown restricted least squares (RLS) estimator is given by 


$$
\hat{\beta}_{R}=\hat{\beta}-\left(X^{\prime} X\right)^{-1} R^{\prime}\left[R\left(X^{\prime} X\right)^{-1} R^{\prime}\right]^{-1}(r-R \hat{\beta})
$$

Let us consider the general linear regression model

$$
Y=X \beta+\varepsilon, \varepsilon \sim\left(0, \sigma^{2} V^{-1}\right)
$$

When $V$ is a known positive definite (p.d.) matrix, the following estimators are proposed:

Aitken (1935) derived the generalized least squares (GLS) estimator as:

$$
\hat{\beta}_{G L S}=\left(X^{\prime} V X\right)^{-1} X^{\prime} V Y
$$

However, in this case the problem of multicollinearity may also arise. So, Trenkler (1984) extended the idea of ridge regression to reduce the effects of autocorrelation and multicollinearity problems and he proposed the generalized ridge (GR) estimator of $\beta$ in the general linear regression model as:

$$
\hat{\beta}_{G R}(k)=\left(X^{\prime} V X+k I_{p}\right)^{-1} X^{\prime} V Y, \quad k>0 .
$$

He concluded that the ridge regression estimator which takes the autocorrelation into account can perform better than some other methods.

Many authors consider some estimation methods for the general linear model in case of $V$ is unknown. So, the feasible generalized restricted least squares (FGRLS) estimator under the prior restrictions in Equation (4) is given by Chaturvedi et al. (2001)

$$
\breve{\beta}_{R}=\breve{\beta}+\left(X^{\prime} \hat{V} X\right)^{-1} R^{\prime}\left[R\left(X^{\prime} \hat{V} X\right)^{-1} R^{\prime}\right]^{-1}(r-R \breve{\beta}),
$$

where $\hat{V}=V(\hat{\theta}), \hat{\theta}$ is a consistent estimator of $\theta$, and

$$
\breve{\beta}=\left(X^{\prime} \hat{V} X\right)^{-1} X^{\hat{V} Y}
$$


is the feasible generalized least squares (FGLS) estimator of $\beta$.Then, the feasible generalized ridge regression (FGR) estimator is given by Eledum and Zahri (2013)

$$
\breve{\beta}_{G R}(k)=\left(X^{\prime} \hat{V} X+k I_{p}\right)^{-1} X^{\prime} \hat{V} Y, k>0 \text {. }
$$

Chaturvedi and Shukla (1990) consider the following SR estimator of $\beta$ :

$$
\check{\beta}_{S}=\left[1-\frac{a}{n} \frac{(Y-X \breve{\beta})^{\prime} \hat{V}(Y-X \breve{\beta})}{\breve{\beta}^{\prime} X^{\prime} \hat{V} X \breve{\beta}}\right] \check{\beta},
$$

where $a \geq 0$ is the shrinkage factor. Replacing $\breve{\beta}$ by $\breve{\beta}_{R}$ in Equation (12), Chaturvedi et al. (2001) considered the following estimator as a restricted SR estimator of $\beta$,

$$
\breve{\beta}_{R S}=\left[1-\frac{a}{n} \frac{\left(Y-X \breve{\beta}_{R}\right)^{\prime} \hat{V}\left(Y-X \breve{\beta}_{R}\right)}{\breve{\beta}_{R}^{\prime} M^{* \prime}\left(X^{\prime} \hat{V} X\right) M^{*} \breve{\beta}_{R}}\right] \breve{\beta}_{R}
$$

where $M^{*}=I-\left(X^{\prime} \hat{V} X\right)^{-1} R^{\prime}\left[R\left(X^{\prime} \hat{V} X\right)^{-1} R^{\prime}\right]^{-1} R$. Moreover, if $\breve{\beta}_{S}$ is replaced for $\breve{\beta}$ in Equation (9), they obtain the restricted estimator,

$$
\bar{\beta}_{R S}=\breve{\beta}_{S}+\left(X^{\prime} \hat{V} X\right)^{-1} R^{\prime}\left[R\left(X^{\prime} \hat{V} X\right)^{-1} R^{\prime}\right]^{-1}\left(r-R \breve{\beta}_{S}\right)
$$

which can be expressed equivalently as,

$$
\bar{\beta}_{R S}=\breve{\beta}_{R}-\frac{a}{n} \frac{(Y-X \breve{\beta})^{\prime} \hat{V}(Y-X \breve{\beta})}{\breve{\beta}^{\prime} X^{\prime} \hat{V} X \breve{\beta}} \hat{S} \breve{\beta}
$$

where $\hat{S}=I_{p}-\left(X^{\prime} \hat{V} X\right)^{-1} R^{\prime}\left[R\left(X^{\prime} \hat{V} X\right)^{-1} R^{\prime}\right]^{-1} R$.

More recently, Özbay et al. (2016) have considered replacing $\breve{\beta}$ by $\breve{\beta}_{G R}(k)$ in Equation (9) and extending the idea of restricted ridge estimation method (see, 
Gross, 2003; Kaçıranlar et al. 2011) for the case of $V$ is unknown, they have defined the feasible generalized restricted ridge regression (FGRR) estimator as follows:

$$
\breve{\beta}_{G R R}(k)=\breve{\beta}_{G R}(k)+\left(X^{\prime} \hat{V} X+k I_{p}\right)^{-1} R^{\prime}\left[R\left(X^{\prime} \hat{V} X+k I_{p}\right)^{-1} R^{\prime}\right]^{-1}\left(r-R \breve{\beta}_{G R}(\mathrm{k})\right)
$$

However, since $\breve{\beta}_{S}$ in Equation (12) depends on the $X^{\prime} \hat{V} X$ matrix, the multicollinearity problem also arises in connection with the FGLS estimator in Equation (10). To reduce the effects of the multicollinearity for the estimators $\breve{\beta}_{s}$, $\breve{\beta}_{R S}$ and $\bar{\beta}_{R S}$, we now propose the following estimators:

Combining the philosophy behind the FGR and SR estimation, if $\breve{\beta}_{G R}(k)$ is replaced for $\breve{\beta}$ in Equation (12), we obtain the following feasible generalized SR ridge (FGSRR) regression estimator:

$$
\breve{\beta}_{G S R}(k)=\left[1-\frac{a}{n} \frac{\left(Y-X \breve{\beta}_{G R}(\mathrm{k})\right)^{\prime} \hat{V}\left(Y-X \breve{\beta}_{G R}(\mathrm{k})\right)}{\breve{\beta}_{G R}^{\prime}(\mathrm{k})\left(X^{\prime} \hat{V} X+k I_{p}\right) \breve{\beta}_{G R}(k)}\right] \breve{\beta}_{G R}(k) .
$$

Following Srivastava and Srivastava (1983, 1984) and Chaturvedi et al. (2001), if we replace $\breve{\beta}_{G R}(k)$ by $\breve{\beta}_{G R R}(k)$ in Equation (17), we obtain the following FGSRR estimator:

$$
\breve{\beta}_{G S R R}(k)=\left[1-\frac{a}{n} \frac{\left(Y-X \breve{\beta}_{G R R}(\mathrm{k})\right)^{\prime} \hat{V}\left(Y-X \breve{\beta}_{G R R}(\mathrm{k})\right)}{\breve{\beta}_{G R R}^{\prime}(\mathrm{k}) M^{* *}\left(X^{\prime} \hat{V} X+k I_{p}\right) M^{* *} \breve{\beta}_{G R R}(k)}\right] \breve{\beta}_{G R R}(k),
$$

where $M^{* *}=I-\left(X^{\prime} \hat{V} X+k I_{p}\right)^{-1} R^{\prime}\left[R\left(X^{\prime} \hat{V} X+k I_{p}\right)^{-1} R^{\prime}\right]^{-1} R$.

If we replace $\breve{\beta}_{G R}(k)$ by $\breve{\beta}_{G S R}(k)$ in Equation (16), we obtain the following FGSRR estimator: 


$$
\bar{\beta}_{G S R R}(k)=\breve{\beta}_{G S R}(k)+\left(X^{\prime} \hat{V} X+k I_{p}\right)^{-1} R^{\prime}\left[R\left(X^{\prime} \hat{V} X+k I_{p}\right)^{-1} R^{\prime}\right]^{-1}\left(r-R \breve{\beta}_{G S R}(\mathrm{k})\right)
$$

Furthermore, our new estimators have some tempting properties. Initially, it is easy to see that the new estimator $\bar{\beta}_{G S R R}(k)$ satisfy the prior restrictions in Equation (4). In fact, from the definitions of the $\breve{\beta}_{G S R}(k)$ and $\breve{\beta}_{G S R R}(k)$, we can see that these estimators are general estimators that include the $\breve{\beta}_{S}$ and $\bar{\beta}_{R S}$ as special cases:

(i) Since $\breve{\beta}_{G R}(0)=\breve{\beta}$, then $\breve{\beta}_{G S R}(0)=\breve{\beta}_{S}$

(ii) Since $\breve{\beta}_{G S R}(0)=\breve{\beta}_{S}$, then $\bar{\beta}_{G S R R}(0)=\bar{\beta}_{R S}$.

\section{THE PMSE UNDER THE TARGET FUNCTION}

In this section, we will represent the prediction performance of our new estimators under the target function. Generally predictions from a linear regression model are made either for the actual values of the study variable or for the average values at a time. However, situations may occur in which one may be required to consider the predictions of both the actual and the average values simultaneously.

If $\tilde{\beta}$ denotes an estimator of $\beta$, then the predictor for the values of study variable within the sample is generally formulated as $\hat{T}=X \tilde{\beta}$ which is used for predicting either the actual values $y$ or the average values $E(y)=X \beta$ at a time. When the situation demands prediction of both the actual and the average values together, the target function is defined as follows,

$$
T(y)=t y+(1-t) E(y)
$$


and use $\hat{T}=X \tilde{\beta}$ for predicting it where $0 \leq t \leq 1$ is a nonstochastic scalar specifying the weightage to be assigned to the prediction of actual and average values of the study variable, see Shalabh (1995), Toutenburg and Shalabh (1996), Toutenburg and Shalabh (2000), Kumar et al. (2008). The PMSE matrix under the target function of the predictor $\hat{T}$ is given by

$$
\operatorname{PMSE}(\hat{T})=E(\hat{T}-T)(\hat{T}-T)^{\prime}
$$

Then, the scalar PMSE under the target function of the predictor $\hat{T}$ is given by

$$
\operatorname{pmse}(\hat{T})=E(\hat{T}-T)^{\prime}(\hat{T}-T)
$$

This strong criterion is used by many authors who are working on the predictive performance of the estimators. Shalabh (1995), Toutenburg and Shalabh (1996, 2000) and Kumar et al. (2008) have theoretically studied the predictive performance of some predictors obtained by using different estimators under the PMSE criterion. They derived some conditions for the superiority of the proposed estimator over the other known estimators.

However, since these conditions depend on the unknown parameters, it is difficult to know how to make use of the theoretical results in practice. Thus, we consider the prediction performance of the estimators $\breve{\beta}_{R}, \breve{\beta}_{G R R}(k), \breve{\beta}_{S}$, $\breve{\beta}_{G S R}(k), \breve{\beta}_{R S}, \breve{\beta}_{G S R R}(k)$ and $\bar{\beta}_{G S R R}(k)$ according to the PMSE criterion under the target function using a Monte Carlo simulation study.

\section{A MONTE CARLO SIMULATION STUDY}

In this section, we will discuss the simulation study to compare the performances of the $\breve{\beta}_{R}, \breve{\beta}_{G R R}(k), \breve{\beta}_{S}, \breve{\beta}_{G S R}(k), \breve{\beta}_{R S}, \breve{\beta}_{G S R R}(k)$ and $\bar{\beta}_{G S R R}(k)$ estimators. MATLAB is used for the simulation experiment. Following 
McDonald and Galarneau (1975) and Kibria (2003), the explanatory variables are generated by

$$
x_{i j}=\left(1-\gamma^{2}\right)^{1 / 2} z_{i j}+\gamma z_{i p+1}, i=1,2, \ldots, n, \quad j=1,2, \ldots, p
$$

where $z_{i j}$ are independent standard normal pseudo-random numbers, $\gamma$ is specified so that the correlation between any two explanatory variables is given by $\gamma^{2}$. Following Kibria (2003), three different sets of correlation are considered, corresponding to $\gamma=0.7,0.8,0.9$. Following Chaturvedi et al. (2001), data are generated using the following orthonormal model with $p=4$ and $n=20$ :

$$
y_{i}=\beta_{1} x_{1 i}+\beta_{2} x_{2 i}+\beta_{3} x_{3 i}+\beta_{4} x_{4 i}+u_{i} ; \quad i=1, \ldots, 20
$$

where the $u_{i}$ 's are assumed to be generated by either an AR(1) process ( $\left.u_{i}=\rho u_{i-1}+\varepsilon_{i}\right)$ or a MA(1) process $\left(u_{i}=\varepsilon_{i}-\rho \varepsilon_{i-1}\right)$; where $\rho$ is taken as $0.8,-0.4,0.0,0.4,0.8$, and $\varepsilon_{i} \sim \operatorname{IN}(0,1)$. Also, $a=p-3$ and $\alpha=2$ are taken like this.

The estimators are compared according to scalar PMSE under target function defined as

$$
\operatorname{pmse}(\hat{T})=\frac{1}{M C N} \sum_{m c i=1}^{M C N}\left(\hat{T}_{m c i}-T\right)^{\prime}\left(\hat{T}_{m c i}-T\right)
$$

where $M C N$ represents the number of Monte Carlo replications which is taken as 5000 .

Following Kaçıranlar et al. (2011), two different types of restrictions are used to see how the number of restrictions affects the pmse values. The first type of restriction about the parameters is taken as $\beta_{1}+\beta_{2}+\cdots+\beta_{p}=\frac{p(p+1)}{2} \times \tau$. For this 
restriction, one may write $R=\left(\begin{array}{lll}1 & 1 & \ldots\end{array}\right)$, and $r=\frac{p(p+1)}{2} \times \tau$. Since the true parameter vector is $\beta=\left(\begin{array}{llll}1 & 2 \ldots p\end{array}\right)^{\prime}$, the sum given in the restriction is equal to $\frac{p(p+1)}{2} \times \tau$.The usage of $\tau$ allows one to control whether the restriction is true or not, and if it not true, to control the difference between the right and left hand sides of the restriction, $\tau$ is selected as $1,1.05$, or 1.10 , and it is fixed constant throughout the replications. Since the left hand side of the restriction is equal to $\frac{p(p+1)}{2}$, it is obvious that the restriction $\beta_{1}+\beta_{2}+\cdots+\beta_{p}=\frac{p(p+1)}{2} \times \tau$ is true when $\tau=1$. However, when $\tau$ is chosen as 1.05 (or 1.10), the right hand side of the restriction is equal to $\frac{p(p+1)}{2} \times 1.05$ (or $\left.\frac{p(p+1)}{2} \times 1.10\right)$, while the left side remains $\frac{p(p+1)}{2}$, which leads to a $5 \%$ (or $10 \%$ ) deviation on the right hand side of the restriction. Since $R, r, \beta$, and $\tau$ are all defined at the beginning of the simulations and fixed through the replications, the restrictions are still nonstochastic. For the second type of restriction $R$ and $r$ are chosen as

$$
R_{\frac{p}{2} \times p}=\left(\begin{array}{ccccccccc}
1 & 1 & 0 & 0 & 0 & 0 & \cdots & 0 & 0 \\
0 & 0 & 1 & 1 & 0 & 0 & \cdots & 0 & 0 \\
\vdots & \vdots & \vdots & \vdots & \vdots & \vdots & \vdots & \vdots & \vdots \\
0 & 0 & 0 & 0 & 0 & 0 & \cdots & 1 & 1
\end{array}\right) \text { and } r_{\frac{p}{2} \times 1}=\left(\begin{array}{c}
3 \\
7 \\
\vdots \\
2 p-1
\end{array}\right) \times \tau
$$

where the number of restrictions is $m=p / 2$. The function and values of $\tau$ are still the same as given in the first restriction.

The approaches of Hoerl et al. (1975) are used to specify the values of $k$. The estimates are denoted as $\hat{k}_{H K}=\frac{\hat{\sigma}_{G L S}^{2}}{\sum_{i=1}^{p} \breve{\beta}_{i}^{2}}, \quad \hat{k}_{H K B}=\frac{p \hat{\sigma}_{G L S}^{2}}{\sum_{i=1}^{p} \breve{\beta}_{i}^{2}}$ and 
$\hat{k}_{L W}=\frac{p \hat{\sigma}_{G L S}^{2}}{\sum_{i=1}^{p} \lambda_{i} \breve{\beta}_{i}^{2}}$ respectively, where $\hat{\sigma}_{G L S}^{2}=\frac{(Y-X \breve{\beta})^{\prime} \hat{V}(Y-X \breve{\beta})}{n-p}$ is the FGLS

estimate of error variance and $\lambda_{i}$ 's are the eigenvalues of the matrix $X^{\prime} \hat{V X}$.

The results of the simulation study are summarized in Tables 1-6.

\section{COMMENTS:}

For Tables 1 and 2, we found the pmse values of the mentioned estimators under Restriction Type 1 when it is true and $\mathrm{AR}(1)$ or MA(1) to study the performance of the mentioned estimators according to $t=0.1,0.5,0.9$ values.

We found the pmse values of the mentioned estimators under Restriction Type 1 and 2 when it is true or not and $\operatorname{AR}(1)$ or $\mathrm{MA}(1)$ according to $t=0.1,0.5,0.9$ values but since there are a lot of tables, we just added the results for $t=0.1$ in Tables 3-6 and for $t=0.5$ and $t=0.9$ are not added.

- When $\rho$ value is increasing, pmse values for all mentioned estimators are decreasing for $t=0.1$. Also, pmse values for all mentioned estimators are decreasing till $\rho$ value equals 0 and then pmse values are increasing till $\rho$ value equals 0.8 for $t=0.5,0.9$.

- When $\gamma$ value is increasing, pmse values for $\breve{\beta}_{S}, \breve{\beta}_{R S}$ and $\breve{\beta}_{R}$ are increasing and decreasing for $\breve{\beta}_{G S R}(k), \breve{\beta}_{G S R R}(k), \breve{\beta}_{G R R}(k)$ and $\bar{\beta}_{G S R R}(k)$ of most of $k$ 's in general.

- For the following pair-wise comparisons among the estimators, in each row the best estimator is highlighted in bold font under each restriction, $\operatorname{AR}(1)$ and MA(1) error term model and $t=0.1,0.5,0.9$. 
1- When we make a comparison between $\breve{\beta}_{S}$ and $\breve{\beta}_{G S R}(k)$ estimators, we have that $\breve{\beta}_{G S R}(k)$ estimator gives better results in pmse values than $\breve{\beta}_{S}$ estimator especially for the estimated value $\hat{k}_{H K B}$ in most cases.

2- When we make a comparison between $\breve{\beta}_{R S}$ and $\breve{\beta}_{G S R R}(k)$ estimators, we have that $\breve{\beta}_{G S R R}(k)$ estimator gives better results in pmse values than $\breve{\beta}_{R S}$ estimator especially for the estimated value $\hat{k}_{H K B}$.

3- When we make a comparison between $\breve{\beta}_{R}$ and $\breve{\beta}_{G R R}(k)$ estimators, we have that $\breve{\beta}_{G R R}(k)$ estimator gives better results in pmse values than $\breve{\beta}_{R}$ estimator especially for the estimated value $\hat{k}_{H K B}$.

4- When we make a comparison between $\breve{\beta}_{R}$ and $\bar{\beta}_{G S R R}(k)$ estimators, we have that $\bar{\beta}_{G S R R}(k)$ estimator gives better results in pmse values than $\breve{\beta}_{R}$ estimator especially for the estimated value $\hat{k}_{H K B}$.

5- When we make a comparison between $\breve{\beta}_{G R R}(k)$ and $\bar{\beta}_{G S R R}(k)$ estimators, we have that $\bar{\beta}_{G S R R}(k)$ estimator gives better results in pmse values than $\breve{\beta}_{G R R}(k)$ estimator especially for the estimated value $\hat{k}_{H K B}$.

- Under the same restriction type, when $t$ value is increasing, pmse values for all mentioned estimators are increasing for the $\mathrm{AR}(1)$ error term model rapidly than for the MA(1) error term model.

- For $\mathrm{AR}(1)$ and $\mathrm{MA}(1)$, the pmse values are so close to each other for all mentioned estimators with the same restriction type and the same value of $t$. 


\section{CONCLUSION}

In this study, we introduce some feasible generalized SR restricted ridge regression estimators to examine multicollinearity and autocorrelation problems simultaneously for the general linear regression model. Then we investigate the performance of the defined estimators using a Monte Carlo simulation study in which the results show that the proposed estimators have smaller pmse values relative to the other mentioned estimators especially for the estimated value $\hat{k}_{H K B}$ in most cases under the first and the second restrictions when they are true or not and when the error term follows $\mathrm{AR}(1)$ or MA(1) model. 


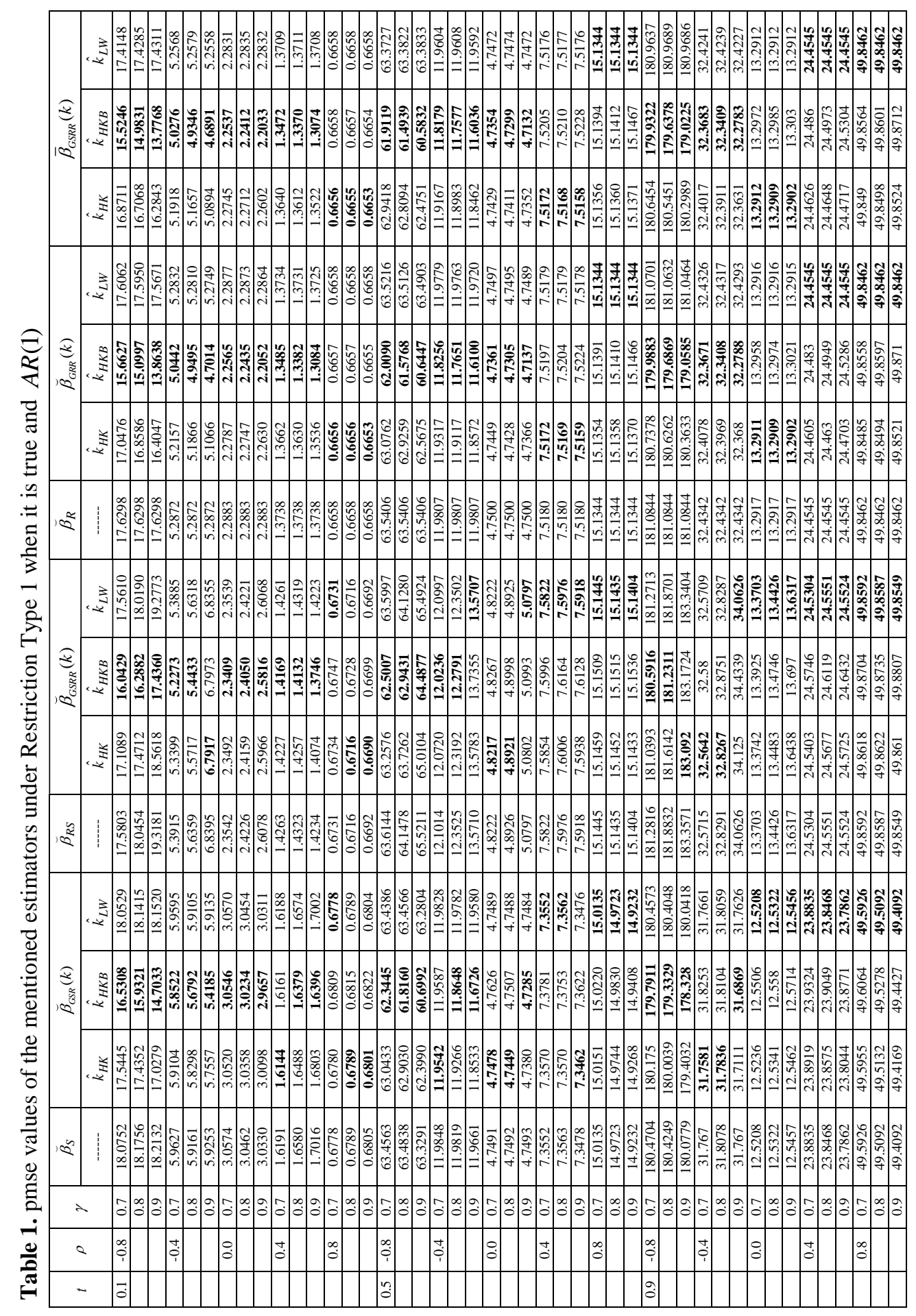




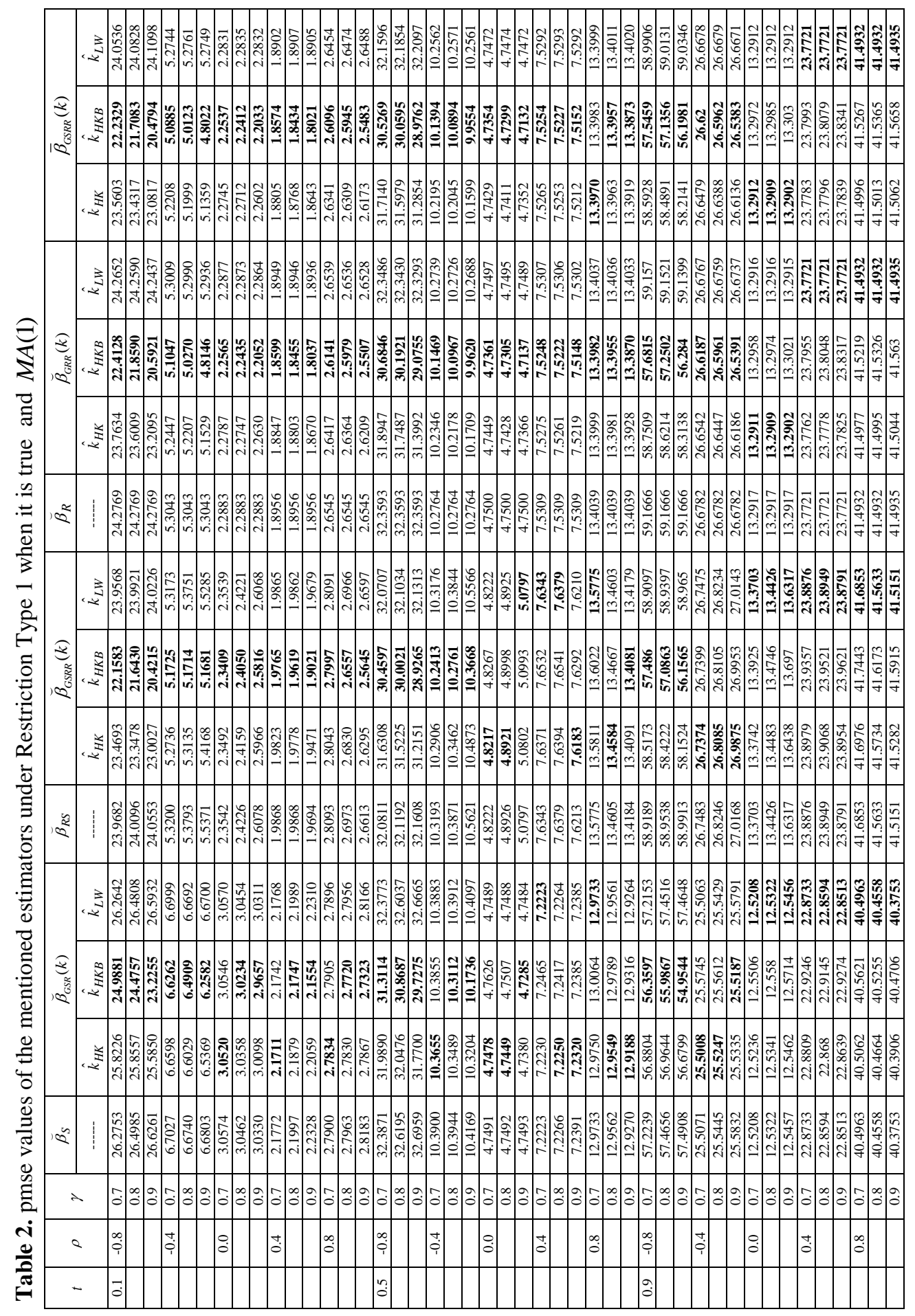




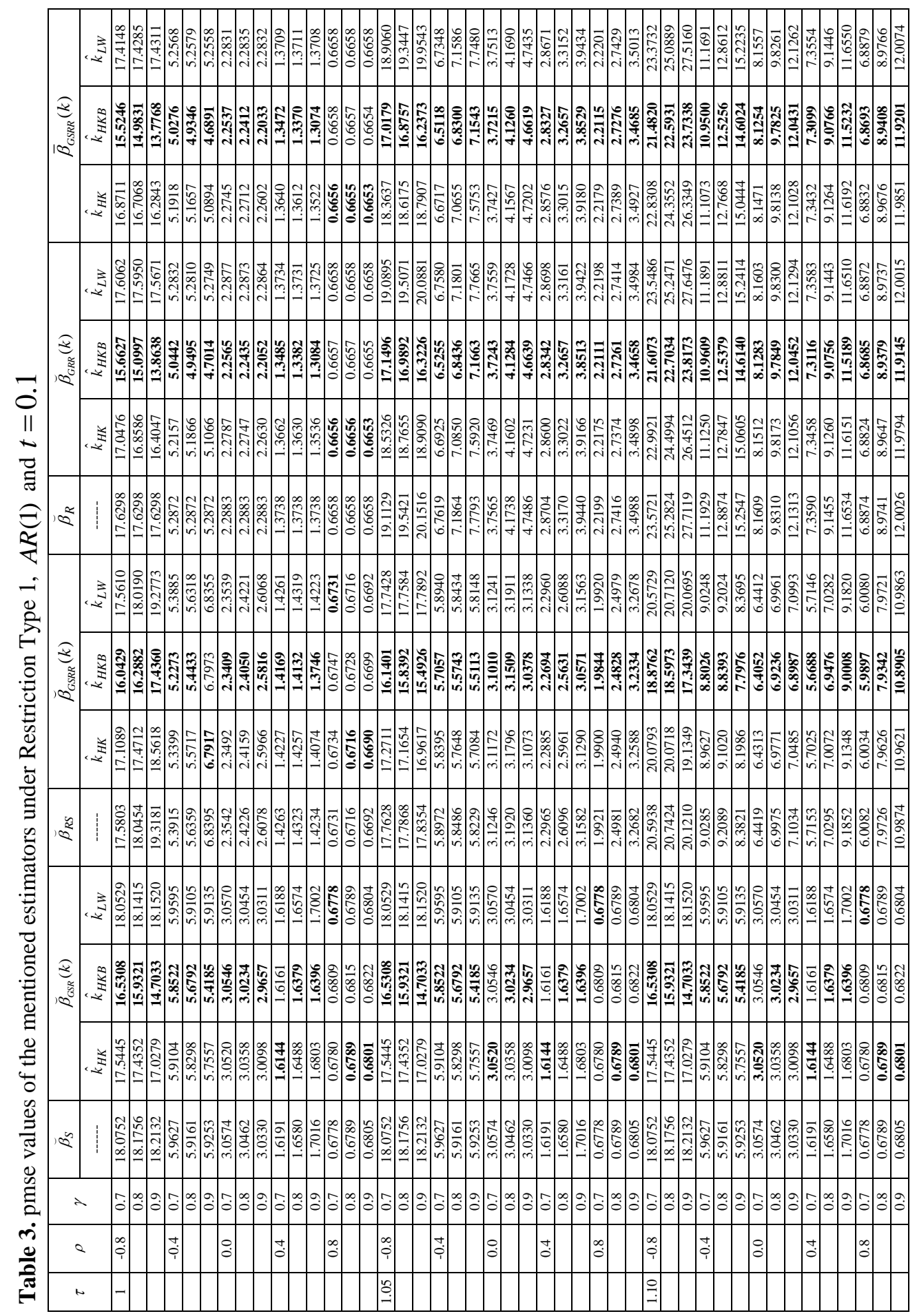




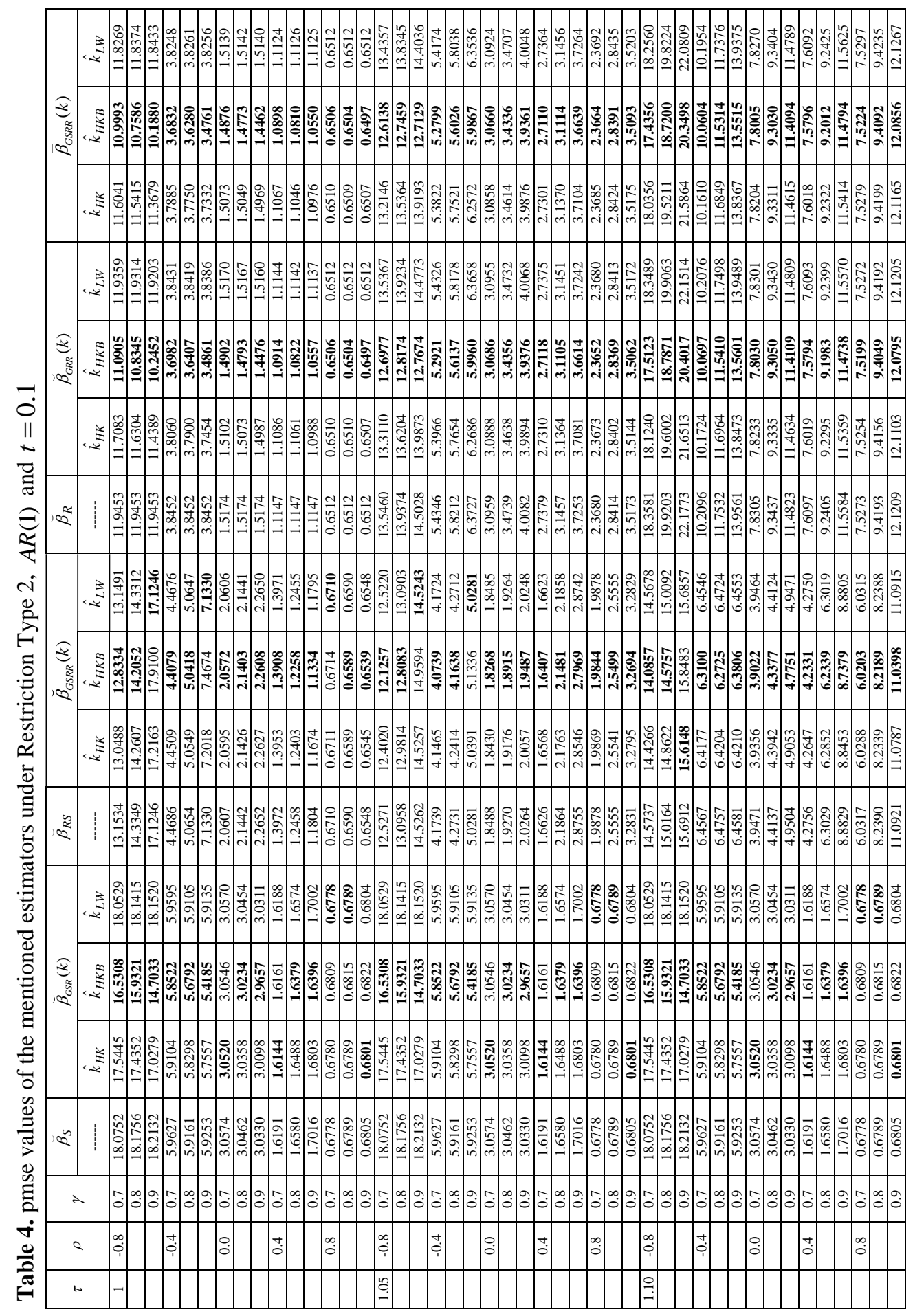




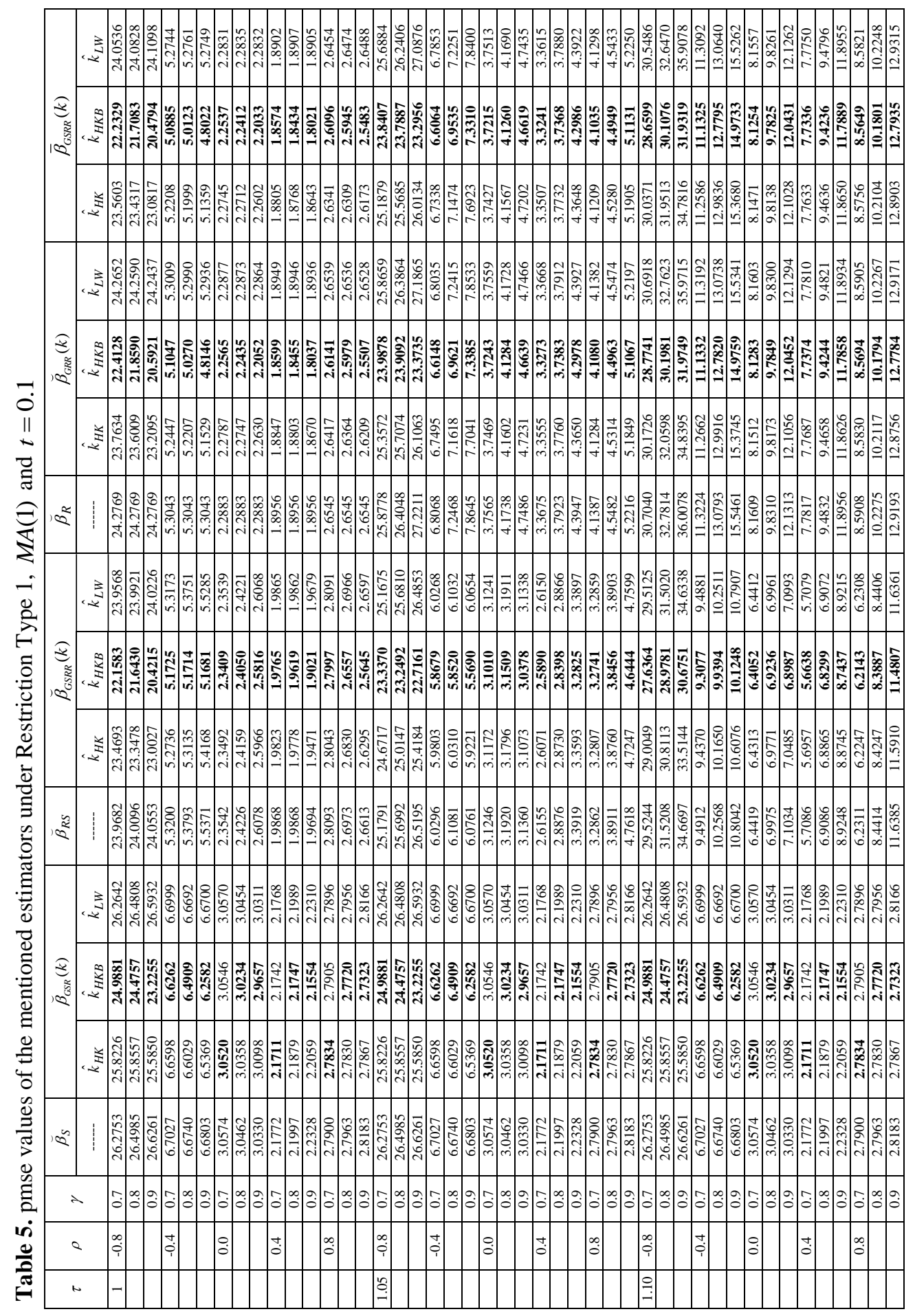




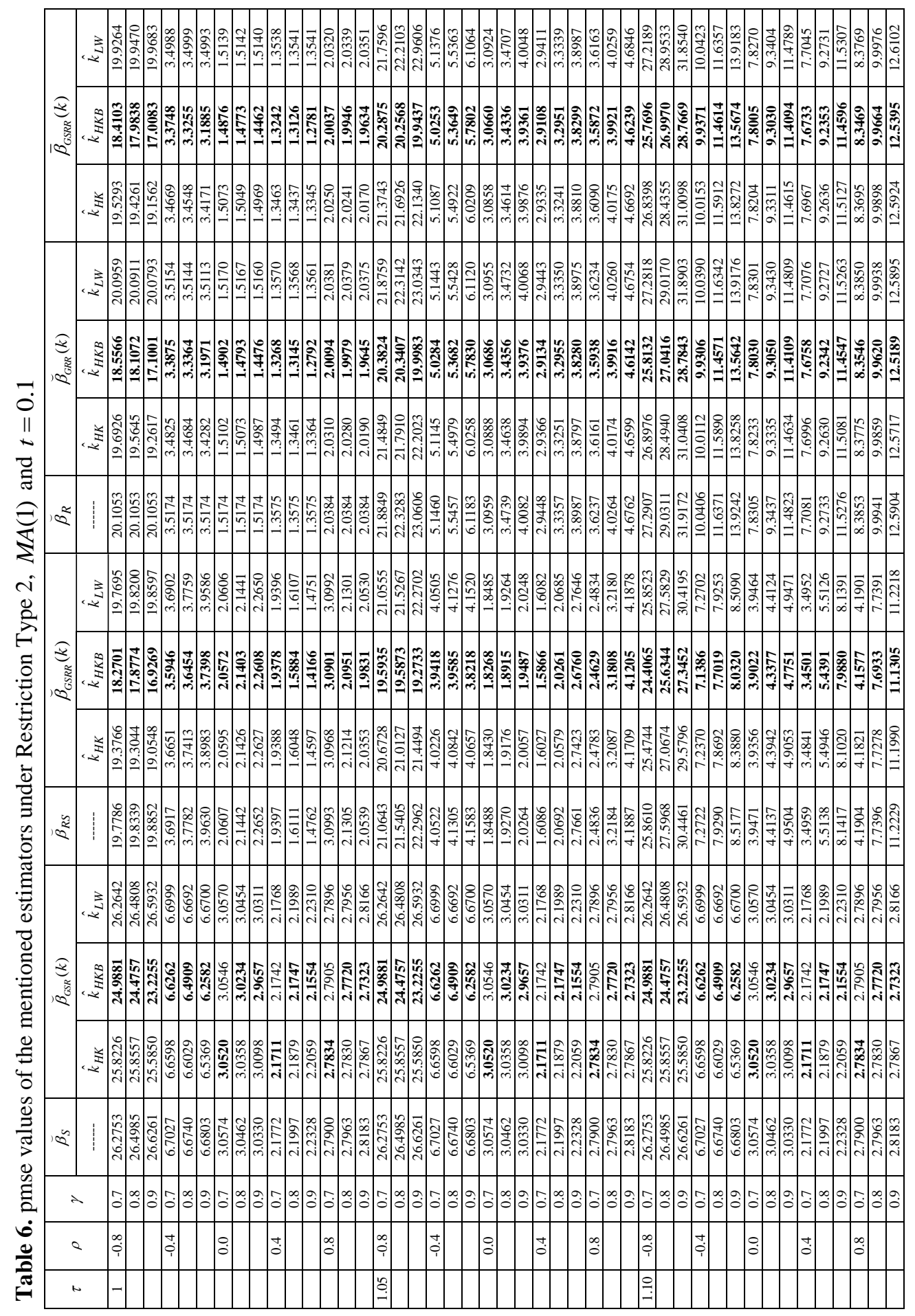




\section{REFERENCES}

Aitken, A.C. (1935). On Least Squares and Linear Combinations of Observations.Proc. Roy. Soc. Edinburgh Sect. A. 55: 42-48.

Chaturvedi, A., Shukla, G. (1990). Stein Rule Estimation in Linear Model with Non-Scalar Error Covariance Matrix.Sankhya B. 52: 293-304.

Chaturvedi, A., Van Hoa, T. and Shukla, G. (1996).Improved Estimation in the Restricted Regression Model with Non-Spherical Disturbances.J. Quant. Econ. 12: 115-123.

Chaturvedi, A., Wan, A.T.K. and Singh, S.P. (2001).Stein-Rule Restricted Regression Estimator in a Linear Regression Model with Nonspherical Disturbances.Comm. Statist. Theory Methods. 30 (1): 55-68.

Chaturvedi, A., Shalabh, S. (2014). Bayesian Estimation of Regression Coefficients Under Extended Balanced Loss Function. Commun.Stat. Theory Methods. 43: 4253-4264.

Eledum, H., Zahri, M. (2013).Relaxation Method for Two Stages Ridge Regression Estimator.Int. J. Pure Appl. Math. Sci. 85 (4): 653-667.

Groß J. (2003). Restricted Ridge Estimation.Statist.Probab.Lett. 65: 57-64.

Güler, H., Kaçıranlar, S. (2009). A Comparison of Mixed and Ridge Estimators of Linear Models.Comm. Statist. Simulation Comput. 38 (2): 368-401.

Hoerl, E., Kennard, R. W. (1970a). Ridge Regression: Biased Estimation for Nonorthogonal Problems. Technometrics 12 (1): 55-67.

Hoerl, A.E., Kennard, R.W. and Baldwin, K.F. (1975). Ridge Regression: Some Simulations. Comm. Statist. Simulation Comput. 4: 105-123.

James, W., Stein, C. (1960).Estimation with Quadratic Loss.Proc. Fourth Berkeley Symp.on Math. Statist.and Prob., Vol. 1, Univ. of Calif. Press, 361-379, Berkeley.

Kaçıranlar, S., Sakallığlu, S., Özkale, M.R. and Güler, H. (2011). More On the Restricted Ridge Regression Estimation. J. Stat. Comput. Simul. 81 (11): 1433-1448.

Kibria, B.M.G. (2003). Performance of Some New Ridge Regression Estimators.Comm. Statist. Simul.Comput. 32 (2): 419-435.

Kumar, M., Mishra, N. and Gupta, R. (2008).Predictive Performance of the Improved Estimators with Exact Restrictions in Linear Regression Models.Amer. J. Math. Management Sci. 28: 419-432.

McDonald, G.C., Galarneau, D.I. (1975). A Monte Carlo Evaluation of Some Ridge Type Estimators.J. Amer. Statist. Assoc. 20: 407-416.

Özbay, N., Kaçıranlar, S. and Dawoud I. (2016).The Feasible Generalized Restricted Ridge Regression Estimator. J. Stat. Comput. Simul. DOI: 10.1080/00949655.2016.1224880.

Shalabh, S. (1995).Performance of Stein-Rule Procedure for Simultaneous Prediction of Actual and Average Values of Study Variable in Linear Regression Model.Bull. Int. Stat. Ins. 56: 1375-1390.

Shalabh, S., Toutenburg, H. and Heumann, C. (2009).Stein-Rule Estimation Under an Extended Balanced Loss Function. J. Stat. Comput. Simul. 79 (10): 1259-1273.

Srivastava, V.K., Srivastava, A.K. (1983). Improved Estimation of Coefficients in Regression Models With Incomplete Prior Information.Biom. J. 25: 775-782.

Srivastava, V.K., Srivastava, A.K. (1984). Stein-Rule Estimators in Restricted Regression Models.Esdadistica. 36: 89-98. 
Srivastava, A.K., Chandra, R. (1991). Improved Estimation of Restricted Regression Model When Disturbances are Not Necessarily Normal. Sankhya, Ser. B. 53: 119-133.

Stein, C. (1956). Inadmissibility of Usual Estimator for the Mean of a Multivariate Normal Distribution.Proc. Third Berkeley Symp.on Math. Statist.and Prob., Vol. 1, Univ. of Calif. Press, 197206, Berkeley.

Toutenburg, H., Shalabh, S. (1996). Predictive Performance of the Methods of Restricted and Mixed Regression Estimators.Biom. J. 38 (8): 951-959.

Toutenburg, H., Shalabh, S. (2000). Improved Predictions in Linear Regression Models with Stochastic Linear Constraints.Biom. J. 42: 71-86.

Trenkler, G. (1984). On the Performance of Biased Estimators in the Linear Regression Model with Correlated or Heteroscedastic Errors. J. Econometrics. 25: 179-190.

Wan, A.T.K., Chaturvedi, A. (2000). Operational Variants of the Minimum Mean Squared Error Estimator in Linear Regression Models with Non-Spherical Disturbances. Ann. Inst. Statist.Math. 52: 332-342.

Nimet Özbay,

Department of Statistics,

Faculty of Science and Letters, Çukurova University, 01330, Adana, Turkey.

Email: nturker@cu.edu.tr

Issam Dawoud,

Department of Statistics,

Faculty of Science and Letters, Çukurova University, 01330, Adana, Turkey.

Selahattin Kaçıranlar, Department of Statistics, Faculty of Science and Letters, Çukurova University, 01330, Adana, Turkey. Email: skacir@cu.edu.tr 